\title{
LA RUTA DEL VINO Y DEL PISCO EN EL VALLE DE VÍTOR (AREQUIPA)
}

\section{THE WINE AND PISCO ROUTE AT THE VITOR VALLEY (AREQUIPA)}

Alejandro Málaga Núñez-Zeballos

Universidad San Agustín de Arequipa, Perú

\section{RESUMEN}

Hace una década se inició la gran difusión de nuestra bebida bandera: el pisco, en diferentes campañas publicitarias a cargo de marcas reconocidas y, sobre todo, a raíz de la creación de la Academia Peruana del Pisco, institución que pone énfasis en los estudios históricos de la noble bebida. En países como España, Portugal, Italia, México, Argentina y Chile se han creado rutas turísticas temáticas a partir de una bebida típica o tradicional, fomentando el flujo de turistas y generando un turismo específico: el enológico. En el Perú, los departamentos de Ica y Moquegua ya cuentan con esta ruta; sin embargo, los valles arequipeños especialmente el de Vítor, con una tradición al respecto desde la segunda mitad del siglo XVI hasta la fechano poseen un circuito turístico que ofrezca este tipo de atractivos. El presente artículo muestra un panorama histórico de las principales bodegas vinícolas y pisqueras del valle de Vítor y propone además la implementación de la Ruta del Vino y del Pisco en dicho valle.

Palabras clave: Bodegas históricas, ruta temática, turismo enológico-pisquero.

\begin{abstract}
A decade ago, a large amount of information on our national drink pisco was made known through different advertising campaigns promoted by renowned brands and especially due to the establishment of the Peruvian Academy of Pisco, institution that emphasizes on historical studies of this fine beverage. In countries such as Spain, Portugal, Italy, Mexico, Argentina and Chile, enological tourist routes have been created based on a typical our traditional beverage, promoting a tourist flow and generating a specific type of tourism: wine tourism. In Peru, Ica and Moquegua regions have already developed this route. However, the valleys in Arequipa, especially Vitor with an enological tradition since the second half of the XVI century to date, do not have a tourist route offering this kind of attractions. This article offers a historical overview of the main wine and pisco cellars of the Vitor valley and moreover, it proposes the implementation of a Wine and Pisco Route in this valley.
\end{abstract}

Key words: Historical wineries, thematic route, pisco and wine tourism 


\section{Antecedentes históricos}

La presencia del vino en Arequipa estuvo inicialmente en las botas de cuero curtido traídas por los españoles que recorrieron por primera vez el valle del río Chili hacia 1535. Luego en la fundación de Arequipa el 15 de agosto de 1540, en la misa y en el brindis de honor, en la ceremonia de la traza de cuadrículas e instalación de la picota, y posteriormente en cada vivienda de las autoridades políticas, religiosas, encomenderos, artesanos y curacas.

En los veinte primeros años de vida, la ciudad estuvo convulsionada por las guerras civiles entre los conquistadores, fueron tiempos en que el flujo de mercaderías procedentes de la península ibérica a los puertos de Quilca y Chule a veces se interrumpía y generaba un alza en los precios. Las principales familias poseían en la margen izquierda del río sus huertas con manzanas, naranjas, membrillos y uvas para consumirse como fruta; la carencia de vino fue suplida por las botijas traídas de Andalucía y Castilla a precios elevados.

El 1 de enero de 1546 llegó un navío al puerto de Quilca con telas de diferentes calidades, camisas, calzas, zapatos, aves, cerdos, caballos, etc., y unas botijas de vino y aceite, que fueron cargadas desde la costa hasta esta ciudad por indios yanaconas enviados por los caciques yarabahas y chilques. Al parecer, no fue buena idea porque el 13 de marzo el Cabildo Civil revocó la ordenanza y prohibió la venta de vino sin previa medida. Siete meses después la autoridad recapacitó y ordenó que los indios fueran a cargar las mercaderías y que los comercializadores les pagaran a los curacas conforme a las ordenanzas establecidas (Archivo Municipal de Arequipa, en adelante AMA, año 1546).

A inicios de 1547 el teniente de capitán Juan de Silveira, a órdenes de Gonzalo Pizarro, estando en Arequipa, ordenó "que los mercaderes se moderasen en el vender el vino los cuales han subido de precio, mandan a rebajar el vino, el más caro de 20 pesos arrobado o rehenchido a contento del que lo comprare e que no lo dexen de vender, ni lo vendan a más precio, so pena de 200 pesos de oro la mitad para la Cámara de su majestad y la otra mitad para el juez que lo sentenciare" (AMA).

Hacia fines de esa década ya existían mesones que brindaban alimentos y vino a propios y viajeros, pero ninguno estaba regulado por un arancel, por ello los precios del vino eran muy dispares y en algunos casos bastante elevados, generando la queja de la ciudadanía; o por el contrario muy baratos, "por menudo y lo venden a esclavos y así mismo que para ver taquies (bailes desenfrenados) de negros en las fiestas, muchas veces se matan unos negros a otros e hacen otras cosas muy feas en servicio de Dios" (AMA). Por ello las autoridades ordenaron que ninguna persona vendiera vino a esclavos si no llevaban una cédula de su amo, "so pena de 10 pesos de oro; que se pregone que ningún esclavo haga taquies en ninguna parte de la ciudad ni fuera de ella y si lo hicieran que el alguacil los prenda e les sean dados 100 azotes, atados a la picota" (AMA). Además, por primera vez se estableció un tarifario de precios para los tambos de Vítor, Siguas, Camaná, Ocoña, Atico, Atiquipa, Acarí, Chule, Chiguata y Quilca; resaltamos el precio de un cuartillo de vino blanco en seis tomines y el de un cuartillo de vino tinto en un peso y dos tomines. 
Hacia 1557, los alrededores de Arequipa eran prósperos terrenos de variados cultivos de maíz, papa, cebolla, trigo y viñedos, que eran constantemente afectados por el ganado que transitaba en desorden; por ello las autoridades ordenaron:

que cualquier ganado ovejuno o puercos o cabras o ganado de la tierra y todo otro ganado menudo que fuere tomado asiendo daño en viñas en esta ciudad y en todos sus términos tenga de pena siendo tomado de día el quinto del dicho ganado siendo de 5 cabezas hasta 50 y den leña del camino se quinte y de noche tenga la pena doblada la cual se aplique las dos partes para el dueño de tal sementera o viña o heredad y la otra parte al juez y obras públicas de la ciudad por mitad (AMA, año 1557).

Claramente, la disposición protege las áreas con vides porque serían las primeras cosechas para elaborar vinos, cuyo comercio en las dos primeras décadas de vida de Arequipa colonial estuvo constituido por importaciones. Posteriormente los mercaderes, al ver su alta rentabilidad, adquirieron tierras para convertirse en productores.

El vino consumido por la población arequipeña desde la fundación de la ciudad hasta mediados de los años cincuenta fue esencialmente traído de Castilla (España); así lo demuestran los manuscritos coloniales. Los miembros de la primera generación de vecinos arequipeños, concretamente los que integraron la élite política, dirigieron el rumbo de sus negocios a la comercialización del vino, ya que se trataba de una inversión muy rentable: más tarde se incrementarían su producción y comercialización. Personas de diferentes estratos sociales y políticos se dedicaron al rubro, dando lugar a un sinfín de situaciones: por ejemplo, en 1565, el mercader Mateo Sánchez, forastero, le adeudaba al tres veces ex alcalde Juan de la Torre la suma de 1.879 pesos y 4 tomines de plata de a 4 pesos el marco por 756 botijas de vino del valle de Pitay, de las cuales 400 embotijadas a precio de 4 pesos de plata y las 356 botijas y sus quintales de pasas a 6 pesos y medio cada quintal (AMA).

En 1568, Diego Hernández de la Cuba, ex alcalde y regidor perpetuo de la ciudad, vendió a Alonso Núñez, clérigo presbítero, toda la cosecha del "vino de la tierra" de la viña y heredad de Lluculla, cada botija a precio de tres pesos de plata corriente. Se compromete a entregarlo en la bodega "a boca de tinaja" en el mes de agosto. Núñez tuvo que conseguir las tinajas para transportar el vino. Ese mismo año Juan del Salto, residente en la ciudad, recibió de Juan de Quiroz Vélez 101 botijas de "vino de la tierra", que sumaron 505 pesos, 20 botijas de vino de Castilla a 10 pesos, suman 200 pesos; y 5 botijas de vinagre, a 6 pesos cada una (AMA N 40: 165).

En 1569 figura una escritura de fletamento entre Diego García Serrano y Bartolomé López, residentes de la ciudad. López fletó a García Serrano 250 botijas de vino blanco de Castilla que tenía en la ciudad y fueron transportadas en "carneros de la tierra" hasta el Cuzco. Partió un 25 de agosto y en el lapso de 40 a 45 días llegó a su destino. Para ese mismo destino, Isidro López vendió a Pedro González 150 botijas de vino en caldo de la cosecha de la heredad de Pitay de Juan de la Torre, puesto en la bodega de Pitay a razón y precio cada botija del vino en caldo a dos pesos y medio de plata corriente. Ese año, la señora Juana Muñiz realizó un contrato por cinco años con el regidor del Cabildo Civil don Diego Cornejo para hacer producir en una heredad de uvas del valle de Vítor, comprando y plantando 50 mil cepas de uvas. Este dato es de suma importancia 
por la elevada cantidad de cepas que iban a ser cultivadas en una enorme extensión, además de indicar a la primera mujer dedicada a esta actividad (AMA).

El cronista Juan López de Velasco, en su Geografía y descripción universal de las Indias, señala acerca de Arequipa: “...el temple de esta ciudad se tiene por el mejor y más apacible de todo el reino del Perú para vivir; llueve en él, y hay invierno y verano como en Castilla, y cógese en su comarca mucho trigo, de que se hace pan muy bueno y sabroso, y gran cantidad de vino, porque las viñas se crían bien en su comarca y se dan mucho á ellas, y el vino se conserva todo lo que quieren” (López de Velasco, 1971: 248).

En 1586, el funcionario de la corona española don Juan de Ulloa y Mogollón consignó: “dáse en esta ciudad gran cantidad de vino, porque hay muchas viñas y se dan mucho a ellas; y tiene cerca de sí un río con su puente" (Ulloa \& Mogollón, 1965: 210).

En su Descripción breve de toda la tierra del Perú, Tucumán, Río de la Plata y Chile, Reginaldo de Lizárraga señala:

Cinco leguas adelante entramos en el valle llamado Vítor; éste es más ancho y donde los más de los vecinos de Arequipa tienen sus heredades; cogen mucho vino y muy bueno, que se lleva al Cuzco y se provee todo el Collao... Volviendo a la ciudad de Arequipa, es del mejor temple de este reino, por estar fundada a la falda de la sierra, de buen cielo, aunque un poco seco; dentro del pueblo se dan muchas uvas y todas las frutas nuestras, en particular peras no mayores que cermeñas... Desde aquí a Arica y aun hasta Chile, ya fenecieron los valles grandes y fértiles y se siguen vallecitos angostos y no de las calidades de los pasados; por eso haremos de ello poca memoria. Desde aquí nos comenzamos a meter la tierra adentro, distante de él 22 leguas y más, en las cuales hay dos valles, uno llamado Ciguas, de muy buena agua y mejor vino. Cinco leguas adelante entramos en el valle llamado Vítor; éste es más ancho y donde los más de los vecinos de Arequipa tienen sus heredades; cogen mucho vino y muy bueno, que se lleva al Cuzco, 65 leguas, y a Potosí, más de 140, y se provee todo el Collao (Lizárraga, 2002: pp. 47-48).

Por su parte, el cronista mestizo Felipe Guamán Poma de Ayala en su Nueva coronica y buen gobierno refiere:

...en la aldea de Acarí, indios de Santiago de Acarí, hanan y hurin, y Uayuri, Palpa; y tiene lo mejor del vino de todo el reino comparado con el vino de Castilla vino dorado clarísimo suave oloroso y de las uvas como mollares y las dichas mollares blanquísimo, tamaño como ciruelas, y tienen trato del vino, de la comida, pan y vino abundancia y poco agua, y tiene carne de sobra, y pasa por ella el camino real para Potosí y Cuzco, Arequipa... (Guamán Poma de Ayala, 1993: 858).

El religioso carmelita Antonio Vázquez de Espinosa, en su Compendio y descripción de las Indias Occidentales, señala que "se cogen más de 100,000 botijas de vino, por ser en este sitio el valle anchuroso donde están plantadas las viñas; tiene buenas caserías y bodegas; para encerrar el vino hornos donde las cuecen tinajas y las botijas" (Vázquez de Espinosa, 1969: 337). 
Todo este auge en la segunda mitad del siglo XVI se vio interrumpido por el desastre natural que tuvo las consecuencias más perjudiciales en toda la historia del sur andino: el 19 de febrero de 1600 Arequipa fue azotada por dos terremotos debido a la erupción del volcán Huaynaputina, además de la lluvia de cenizas que durante 15 días cayó sobre la ciudad, reducciones indígenas y campos de cultivo, diezmando todo (Málaga, 2002).

Durante los siglos XVII, XVIII y gran parte del XIX, el valle de Vítor siguió produciendo miles de botijas de vino y aguardiente, pero bajó su producción por la crisis minera en Potosí y los cambios políticos que afectaron intereses particulares. En algunos casos los agricultores cambiaron las vides por arroz o por caña de azúcar, productos mucho más rentables y de menor cuidado que las ancestrales cepas coloniales; otros mantuvieron a duras penas sus viñedos y llegaron hasta nuestros días con sus productos artesanales que ahora son preciados vinos y piscos que entran en la modernidad de la producción y competencia nacional.

\section{Metodología}

En la parte metodológica de esta investigación se plantea el problema de la siguiente manera: ¿el valle de Vítor cuenta con las características necesarias para la creación de la ruta temática del vino y del pisco?

El objetivo general es determinar el potencial turístico del mencionado valle para la creación de esta ruta temática, y los objetivos específicos son estudiar las características de sus principales bodegas y elaborar la Ruta del Vino y del Pisco en dicho valle. Las preguntas de la investigación son: ¿el valle posee los atractivos turísticos y la infraestructura necesarias para recibir turistas?, ¿cuáles son las bodegas que cuentan con una adecuada infraestructura para integrarse a la Ruta del Vino y del Pisco?

La presente investigación se justifica ya que la ruta turística propuesta articula a las principales bodegas en la primera Ruta del Vino y del Pisco del departamento de Arequipa, generando ingresos para los productores que incrementarán sus ventas y posiblemente llegarán a exportar sus productos. Será la primera ruta temática de la región.

Para la creación de la ruta temática propuesta se visitarán todas las bodegas del valle de Vítor, con el fin de elaborar un catastro y elegir posteriormente las adecuadas para ser incluidas en la ruta. Con el catastro se podrá tener un panorama real de las bodegas abandonadas, de las pequeñas bodegas artesanales y de las bodegas artesanales-industriales. Además, se aplicará un cuestionario para establecimientos cuyos productos van a ser difundidos a partir de la creación de una ruta temática turística.

En el Perú, la Norma Técnica emitida por el Consejo Regulador de la Producción del Pisco indica que solo los departamentos de Lima, Ica, Arequipa, Moquegua y Tacna pueden ser considerados como productores oficiales, ya que históricamente los valles de estos departamentos fueron y son los principales productores de la bebida bandera del Perú; además de una singular y numerosa variedad de vinos. 
Lima, Ica y Moquegua ya cuentan con la propuesta de una ruta temática como una alternativa innovadora para el desarrollo del turismo enológico y pisquero, y para atraer más visitantes a cada valle, que degusten y adquieran los productos derivados de las cepas de uvas históricas, muchas de ellas existentes desde la llegada de los españoles.

El valle de Vítor, a media hora de camino de Arequipa por la carretera Panamericana Sur, fue el principal productor de vinos y aguardientes de uva desde fines del siglo XVI hasta mediados del siglo XVIII en el virreinato peruano. Posee un acervo histórico y cultural, ya que hasta la fecha se conservan centenarias bodegas y tinajas, además de las técnicas ancestrales de procesamiento de los mostos y jugos para convertirlos en vinos y piscos de diferentes tipologías.

Con toda esta riqueza enológica y pisquera, a la fecha no se ha articulado ninguna propuesta como la presente, de allí su atractivo y singularidad. Se plantea poner en valor las bodegas y sus productos, generando un tipo de turismo -enológico y pisquero- que a la fecha está cobrando mucha importancia. Claros ejemplos los vemos en España (Rioja, Castilla-La Mancha, Andalucía, etc.), en Argentina (Córdova, Mendoza, etc.) y en Chile. Con el desarrollo de la Ruta del Vino y del Pisco en Vítor se tendrá una mayor afluencia de turistas a esa zona, además de generar una conciencia turística en los pobladores locales y en los de la Región Arequipa.

El valle de Vítor posee las bodegas más antiguas del sur del Perú, algunas de las cuales datan de la segunda mitad del siglo XVI. Testimonio de ello lo dan las centenarias tinajas y alambiques que llegaron a producir miles de litros para abastecer un área que abarcaba desde Arequipa y el altiplano hasta Potosí.

La presente investigación sobre la creación de la Ruta del Vino y del Pisco en el histórico valle de Vítor del departamento de Arequipa se apoya en los cuestionarios que se aplicarán a los dueños de las bodegas para tener una ficha completa de cada una de ellas. En dicha ficha se consignará una cantidad de datos que permitan apreciar si el establecimiento posee condiciones para ser integrado a la ruta temática, agrupando a las históricas bodegas de este valle en el primer circuito enológico y pisquero del sur del Perú, beneficiando a los productores de vinos y piscos.

La hipótesis planteada es la siguiente: es probable que el valle de Vítor cuente con las características necesarias para la creación de una ruta temática del vino y del pisco. Las tres variables manejadas son: las bodegas, porque se elaborará un directorio de ellas; el producto, porque se verán las diferentes variedades de vinos y piscos; la ruta, que recorrerá ambas márgenes del río.

Se entiende por turismo enológico el flujo de visitantes que arriban a un lugar específico para degustar los vinos en sus diferentes variedades, además de disfrutar visitando las bodegas y los viñedos. En España las zonas vinícolas son muy prestigiosas, igual que las denominaciones de origen en las distintas regiones como Galicia, Castilla y León, Navarra, La Rioja, Penedés, Priorato, La Mancha, Levante, Ronda, Jerez y Ribera del Duero. Entre las ciudades que se distinguen por sus vinos figuran Barcelona, Madrid, Bilbao y Burgos ${ }^{1}$.

1 http://blog.vinos.com/bodegas-vinedos/turismo-enologico/ 
Para el diseño de la ruta se debe elegir un lugar adecuado para emplazarla, realizar un inventario de los recursos locales, definir la longitud del recorrido considerando el tiempo del que disponen los visitantes, seleccionar los rasgos interpretativos que cubran suficientemente el tema y mantenerlos en buen estado, trabajar con los posibles tópicos de la ruta, verificar los puntos de interés con relación al tema general, diseñar el itinerario, tipo de guiado, seguridad del visitante y evaluar el impacto del entorno ${ }^{2}$.

\section{Las bodegas}

Nuestro universo son las 30 bodegas el valle de Vítor, agrupadas sobre la base de la información del Comité de Productores de Vinos y Piscos de Vítor y de sus propios habitantes.

Bodega abandonada en Pueblo Nuevo

\section{Cáceres}

Casa de Sillar

Dos Calaveritas

El Chañal

El Chocho

El Fundador

El Socavón

Fundo La Rodríguez

Fundo $\mathrm{N}^{\circ} 2$

Fundo $\mathrm{N}^{\circ} 3$

Fundo $N^{\circ} 4$

Hacienda del Monte

Hacienda perdida Pueblo de los Desamparados

Jorge Estremadoiro

La Calderón Grande al pie de la cuesta

La Canduelos

La Compañía

La Velarde Grande

Los Sauces

Manuel Arce 1910

Paz-Soldán

Postigo

2 www.utntyh.com/wp-content/uploads/2012/04/Rutas.docx 
San Juan

Santa Teresa

Santuario

Sendero

Torres de la Gala

Viña Vítor

Zegarra e hijos

Las cinco bodegas que producen vino y pisco en el valle de Vítor y que han sido consideradas en el Directorio Nacional de Productores de Pisco del Ministerio de la Producción (Produce) 2008, son las siguientes:

\section{El Fundador}

Marca: $\quad$ Postigo

Representante: Ana Postigo de Castro

Dirección: $\quad$ La Caleta s/n, Vítor, Arequipa. Urb. La Cantuta C-5 José Luis Bustamante y Rivero

Teléfono: (054) 465-594

Mail:_ piscopostigo@gmail.com / piscopostigo@hotmail.com

\section{El Socavón}

Marca: $\quad$ El Socavón

Representante: Joniu Chávez Lucero

Dirección: $\quad$ Calle Sucre 535, Cercado, Vítor, Arequipa

Teléfonos: (054) 224-100 / (054) 465-767

Mail: $\quad$ jchavez_182@hotmail.com

\section{Los Sauces}

Marca: $\quad$ Orgullo Perú

Representantes:Félix Callachi y Verónica Salazar

Dirección: Pueblo Nuevo Altura Km. 1 Carretera Panamericana, Vítor, Arequipa Urb. Campo de Marte Mz. H, Lote 13. Comité 4, Paucarpata

Teléfono: (054) 467-353

Mail:_focus_up@hotmail.com/pisco_orgulloperu@yahoo.com 


\section{Viña Vítor}

Marca: Pisco Viña Vítor

Representante: Roger Carpio Chambi

Dirección: Vía Panamericana 110 - Valle de Vítor, Vítor, Arequipa

Ricardo Palma 307, Urb. California, Paucarpata

Teléfonos: (054) 959-742-084 / (054) 462-956

\section{Zegarra e Hijos}

Marca: $\quad$ La Hacienda del Abuelo

Representante: José Santos Zegarra Bernal y Julio César Zegarra Ríos

Dirección: Urb. Dolores B-8 J. L. Bustamante, Vítor, Arequipa

Jr. Jesús Vera Fernández 877, Urb. San Germán, Lima 31

Teléfonos: (054) 422-224

Hay seis bodegas que no figuran en los directorios mencionados, pero los dueños están dispuestos a integrarse a la ruta y cuentan con infraestructura apropiada:

Fundo $\mathrm{N}^{\circ} 2$

Fundo $\mathrm{N}^{\circ} 3$

Hacienda El Chañal

Jorge Estremadoiro

Postigo

Torres de la Gala

Hay diez bodegas que producen vino y pisco en el valle de Vítor y que no integrarían la ruta turística por su infraestructura y desinterés de los dueños:

Cáceres

El Chocho

Fundo $\mathrm{N}^{\circ} 4$

La Calderón

La Canduelos

La Rodríguez

Paz-Soldán

San Juan

Santa Teresa

Sendero 
Hay ocho bodegas abandonadas:

Bodega abandonada en Pueblo Nuevo

Bodega Casa de Sillar

Dos Calaveritas

Hacienda del Monte

Hacienda perdida Pueblo de los Desamparados

La Compañía

La Velarde Grande 1910

Santuario

De todos los listados se han escogido cuatro bodegas con condiciones para integrar la ruta propuesta. Figuran en el directorio de productores de pisco de Produce y al visitarlas pudimos ver que cuentan con instalaciones cómodas y con los mínimos servicios para poder ser visitadas; además de la intención e interés de sus propietarios por difundir sus variados productos de diferentes calidades. Ellas son: El Chañal, El Socavón, Viña Vítor y Zegarra; asimismo, se podría incluir en la visita la bodega abandonada de los jesuitas llamada La Compañía, pues conserva una iglesia, claustros, lagar y bodega.

\section{Consideraciones finales}

La presente propuesta de creación de una ruta temática, en este caso enológica y pisquera, se basa en la necesidad de tener un producto turístico que represente a la Región Arequipa, ya que no cuenta con ella.

Con la base de datos de las bodegas perfilamos las que serán seleccionadas por sus atributos y productos de calidad con Denominación de Origen, para que puedan competir con similares de otros departamentos.

La puesta en marcha de la ruta temática dependerá del Municipio de Vítor, para poder implementar el camino con la adecuada señalización, así como coordinar las charlas de sensibilización a los bodegueros y a la población en general en buenas prácticas turísticas.

Los turistas en busca de nuevos sabores del vino y del pisco arequipeño tendrán oportunidad de visitar cinco bodegas, apreciando el valle de Vítor y su campiña, además de generar recursos a otros pobladores en sus pequeños negocios como tiendas y restaurantes. 


\section{Referencias}

Academia Peruana del Pisco (2009). Boletín de la Academia Peruana del Pisco, $\mathrm{N}^{\circ} 1$.

Lima. Academia Peruana del Pisco (2011). Boletín de la Academia Peruana del Pisco, $\mathrm{N}^{\circ}$

2. Lima. Academia Peruana del Pisco (2013). Boletín de la Academia Peruana del Pisco,

$\mathrm{N}^{\circ}$ 3. Lima. Ángeles, C. (1972). Peruanidad del pisco. Lima: Editorial Nueva Educación.

Brown, K. (2008). Borbones y aguardiente. La reforma imperial en el sur peruano: Arequipa en vísperas de la Independencia. Lima: Banco Central de Reserva del Perú e Instituto de Estudios Peruanos.

Buller, C. (2011). Vinos, aguardiente y mercado. Auge y declive de la economía del vino en los valles de Arequipa (1770-1853). Lima: Quellca, Centro de Estudios Andinos.

Guamán Poma de Ayala, F. (1993). Nueva coronica y buen gobierno. México-Lima: Fondo de Cultura Económica.

Huertas, L. (2008). Cronología de la producción del vino y del pisco. Perú: 1548-2008. Lima: Academia Peruana del Pisco y Universidad Ricardo Palma.

Lizárraga, Reginaldo de (2002). Descripción del Perú, Tucumán, Río de la Plata y Chile. Madrid: Dastin, D.

L. López de Velasco, J. (1971). Geografía y descripción universal de las Indias. Madrid: Ediciones Atlas.

López-Guzmán, T. \& Sánchez, S. (2008). La creación de productos turísticos utilizando rutas enológicas. Pasos, 6(2), $159-171$.

Málaga, A. (2002). El enojo de los dioses. Terremotos y erupciones en Arequipa del siglo XVI. En Homenaje a Franklin Pease G. Y. Lima: Pontificia Universidad Católica del Perú y Banco de Crédito del Perú.

Martorell, A. (2010). Itinerarios culturales y patrimonio mundial. Lima: Universidad de San Martín de

Porres. Norma Técnica Peruana (2006). NTP211.001 Lima: Indecopi.

Peña, E. de la (2006). Vinos \& Licores. Lima: Mirbert.

Ulloa y Mogollón, J. (1965). Descripción de las Indias que su Magestad manda hacer de 1586. Madrid: Ediciones

Atlas. Vázquez de Espinosa, A. (1969). Compendio y descripción de las Indias Occidentales. Madrid: Ediciones Atlas.

Vera, G. (2010). Lima. El Piscosauer y el Morris Bar. Para una historia del pisco en el Perú. Lima: Sociedad y

Comunicación. Wiesenthal, M. \& Navarro, F. (2010). Enciclopedia del vino. Lima: Producciones Cantabria. 